\title{
Reduced field Scenario with X3 heating in W7-X
}

\author{
Nikolai B. Marushchenko ${ }^{1, *}$, Pavel Aleynikov ${ }^{1}$, Craig D. Beidler ${ }^{1}$, Andreas Dinklage ${ }^{1}$, Joachim Geiger $^{1}$, Per Helander $^{1}$, \\ Heinrich P. Laqua ${ }^{1}$, Henning Maassberg ${ }^{1}$, Yuri Turkin ${ }^{1}$, and the W7-X Team \\ ${ }^{1}$ Max Planck Institute of Plasma Physics, EURATOM Association, Wendelsteinstr. 1, Greifswald, DE17491, Germany
}

\begin{abstract}
In the present work, an ECRH scenario with reduced magnetic field $1.75 \mathrm{~T}$ is considered. For $140 \mathrm{GHz}$, this field corresponds to X3 heating. The high mirror-ratio magnetic configuration, $B_{01} / B_{00} \simeq 0.24$, was considered as one from most attractive for long-pulse operation with low bootstrap current. Since X3 wave mode can be effectively absorbed only in sufficiently hot plasmas, a preheating stage is necessary, and the requirements for target plasmas suitable for starting X3 have been studied. Different ways to establish target plasmas are also discussed, in particular, augmenting X3 heating with X2 beams at $105 \mathrm{GHz}$.
\end{abstract}

\section{Introduction}

At the near-term focus of the W7-X scientific program are experiments to help assess stellarator optimization in view of economic operation of a stellarator fusion power plant [1]. The high-level scientific goals of W7-X are the demonstration of improved neoclassical confinement as well as improved confinement of fast ions, plasma stability up to a volume-averaged $\beta$ of $5 \%$, and a stiff magnetic equilibrium to facilitate the island divertor concept [2] while achieving steady-state operation.

W7-X is equipped with 10 gyrotrons, which generate RF beams of $140 \mathrm{GHz}$ with power up to $1 \mathrm{MW}$ or (optionally) of $105 \mathrm{GHz}$ with $0.5 \mathrm{MW}$ each, respectively [3, 4]. The quasioptical system in W7-X has been well tested for the main frequency, $140 \mathrm{GHz}$, and operation with different frequencies requires an additional adaptation. Scenarios with $105 \mathrm{GHz}$ however have been included in the list of possible options.

The main ECRH scenarios are designed for operation at $B_{0} \simeq 2.5 \mathrm{~T}$ and $140 \mathrm{GHz}$ with the X2-mode for moderate density, $n_{e}<1.2 \times 10^{20} \mathrm{~m}^{-3}$, and $\mathrm{O} 2$ - mode for high density, $n_{e}<2 \times 10^{20} \mathrm{~m}^{-3}$. The launch-ports are close to the "bean-shaped" poloidal plane of the W7-X magnetic configuration, where the maximum of $B$ is located and the radial dependence of $B$ is tokamak-like. Additionally, two remote steering launchers (RSL) are installed near the triangular plane, i.e. near the minimum of $B$.

Already during the initial experimental campaigns, the main ECRH/ECCD scenarios have been well tested [5]. In particular, the possibility of heating with a pure multipass $\mathrm{O} 2$-mode scenario was demonstrated. This scenario was performed in three steps: start-up by $\mathrm{X} 2$, then adding the $\mathrm{O} 2$ beams and, finally, ramping-up the density and switching off the X2 beams. High power ECRH heating with multipass $\mathrm{O} 2$ beams has been performed due to a set of Tungsten-coated holographic tiles installed on the

*e-mail: nikolai.marushchenko@ipp.mpg.de wall opposite to the ECRH launchers for refracting back about $90 \%$ of power [6]. For the same purpose, a nonholographic polished steel liner installed on the outboard side.

Several magnetic configurations were tested, including the high-mirror configuration with a mirror ratio of about $10 \%$. At the same time, configurations with mirror ratios between $15 \%$ and $24 \%$, which have specific relevance as candidates for long-pulse operation due to their small bootstrap currents [7], exceed present technical limits at nominal field, $2.5 \mathrm{~T}$ [8], and were not cleared for operation in the nearest experimental campaigns. However, for scenarios with reduced magnetic field these configurations can be applied. The reduction of the field also opens an additional option for experiments at high- $\beta$ plasmas addressing fundamental MHD predictions and ECRH with the $\mathrm{X} 3$-mode has to be considered as the primary option.

Applications of the X3-mode in W7-X was considered for different goals already; see, for example, the references [3] and [9] (in the last, $\mathrm{X} 3$ at $140 \mathrm{GHz}$ launched near the minimum of $B$ in high-mirror configuration was investigated with respect to selective heating of trapped/passing electrons). Similarly to $\mathrm{O} 2$ scenarios, $\mathrm{X} 3$ heating cannot be used for start-up. It is therefore anticipated that the scenario with X3-mode first requires the establishment of a target plasma with density and temperature required for stable X3 operation. An important task is to find the general requirements for such target plasmas.

At the moment, three possible methods are under consideration for the role of creating the target-plasma: NBI, ICRH and ECRH with X2 at the frequency $105 \mathrm{GHz}$. The assessment of plasma start-up with pure NBI in W7-X was investigated already in detail in Ref. [15]. It was found that the time for target-plasma establishment is too long (about $4 \mathrm{~s}$ ) and even exceeds the time of NBI operation. Investigation of ICRH start-up and creating the target-plasma needs to be done. 

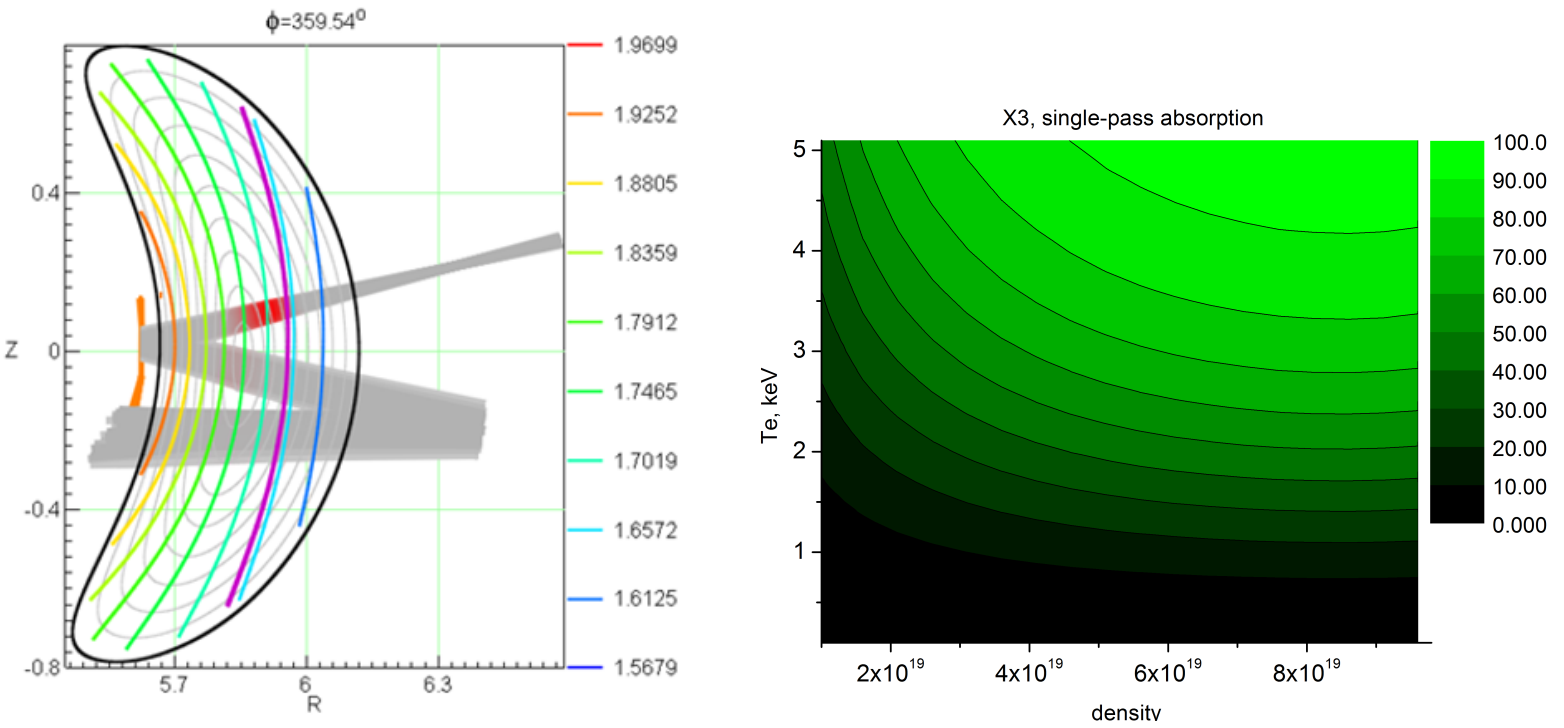

Figure 1. Left: $R-Z$ projection of the RF beam that performs X3 (140 GHz) heating. Three passes are shown, with reflections from the mirror and vacuum chamber. Right: For the beam at the left, a single-pass absorption of the X3- mode is shown as a function of density and electron temperature.

In this paper we analyze the conditions necessary for stable operation of $\mathrm{X} 3$ heating in the magnetic configurations with very high mirror ratios and consider the mixed X2 (105 GHz) and X3 (140 GHz) ECRH scenarios. All simulations are performed with the ray-tracing code TRAVIS [10] coupled to the transport code NTSS [11].

\section{$2 \times 3$ heating and requirements for target plasmas in W7-X}

In order to obtain initial information about the effectiveness of X3 ECRH at $140 \mathrm{GHz}$, the cyclotron absorption must be scanned over plasma parameters. For this purpose, the magnetic configuration with highest mirror ratio, $B_{01} / B_{00}=24 \%$, designated UEM, was applied, and the magnetic field magnitude, $B_{0}(0)=1.75 \mathrm{~T}$, was adjusted to maximize the deposition near the axis. Typically, plasmas in W7-X are optically gray for the X3-mode and several passes are required for complete absorption of the RF beam. Fig. 1 (left) shows the geometry of the X3-beam launched through the port near the "bean-shaped" plane.

In order to control the deposition profile and to avoid any damage for the reflecting mirror from the $1 \mathrm{MW}$ beam, the single-pass absorption for X3-mode in steady-state must exceed $70 \%$. Fig. 1 (right) shows the fraction of absorbed heating power from which one can see that the lowdensity range, $(1-5) \times 10^{19} \mathrm{~m}^{-3}$, seems to be less preferable for X3-mode since quite high temperature, $T_{e} \gtrsim 3 \mathrm{keV}$, appears to be required for sufficient microwave absorption. However, since the plasma heating leads to better absorption when the temperature increases, a more refined analysis has been conducted taking into account the dynamics of the electron temperature into account starting from very small values of it.

To this end, we perform transport simulations (NTSS + TRAVIS) for scenarios at a plasma density range
$(1-5) \times 10^{19} \mathrm{~m}^{-3}\left(n_{e}\right.$-profile fixed $)$ and $5 \mathrm{MW}$ X3 heating from six RF-beams with launch geometry similar to that shown in Fig. 1 (left). It is assumed that neoclassical transport is dominant within the bulk plasma, while the periphery is characterized by anomalous transport proportional to $1 / n_{e}$. The neoclassical transport coefficients were calculated by the DKES code [12]. Simulations were performed as follows: it was assumed that the target plasma had been created in some manner and only the initial density and temperatures, $n_{e}(0)$ and $T_{e, i}(0)$, were changed from run to run. For every RF beam, three passes were taken into account and for rather low $T_{e}$ only a small fraction of power was absorbed. Simulations yielding very low absorption are not of interest. Actually, we select only the cases with a rapid electron temperature growth and omit other cases. If the growth of $T_{e}$ appears, absorption of the X3-mode will increase as it is proportional to $n_{e} T_{e}^{2}$. A plasma build-up time is defined as the characteristic time interval $\Delta t_{1 / 2}$ for which $P_{\mathrm{abs}} \geqslant P_{\text {inj }} / 2$ is reached. Further evolution of the plasma parameters in the phase $t>\Delta t_{1 / 2}$ is usually very rapid as a physical process, but sometimes requires rather time consuming calculations.

In Fig. 2 (left), $\Delta t_{1 / 2}$ is plotted as a function of the initial $n_{e}(0)$ and $T_{e}(0)$ values. As a margin for acceptability of the target plasmas parameters, which comes from safety requirements for the reflecting mirrors, $\Delta t_{1 / 2}=0.1 \mathrm{~s}$ (shown by red) is chosen. The domain with $\Delta t_{1 / 2}>0.1 \mathrm{~s}$ which lies in Fig. 2 (left) below this line, is qualified as not acceptable. It is found that the minimum start-up criterion, $\Delta t_{1 / 2}<0.1 \mathrm{~s}$, is practically equivalent to the requirement $T_{e}>0.7 \mathrm{keV}$ for target plasmas.

It is necessary to mention here that if establishment of a hot plasma occurs, the final steady state $\left(t \gg \Delta t_{1 / 2}\right.$ and $P_{\text {abs }} \simeq P_{\text {inj }}$ ) found by simulations for the given density is practically independent of the initial temperatures. In Fig. 2 (right), an example of the steady-state is shown 

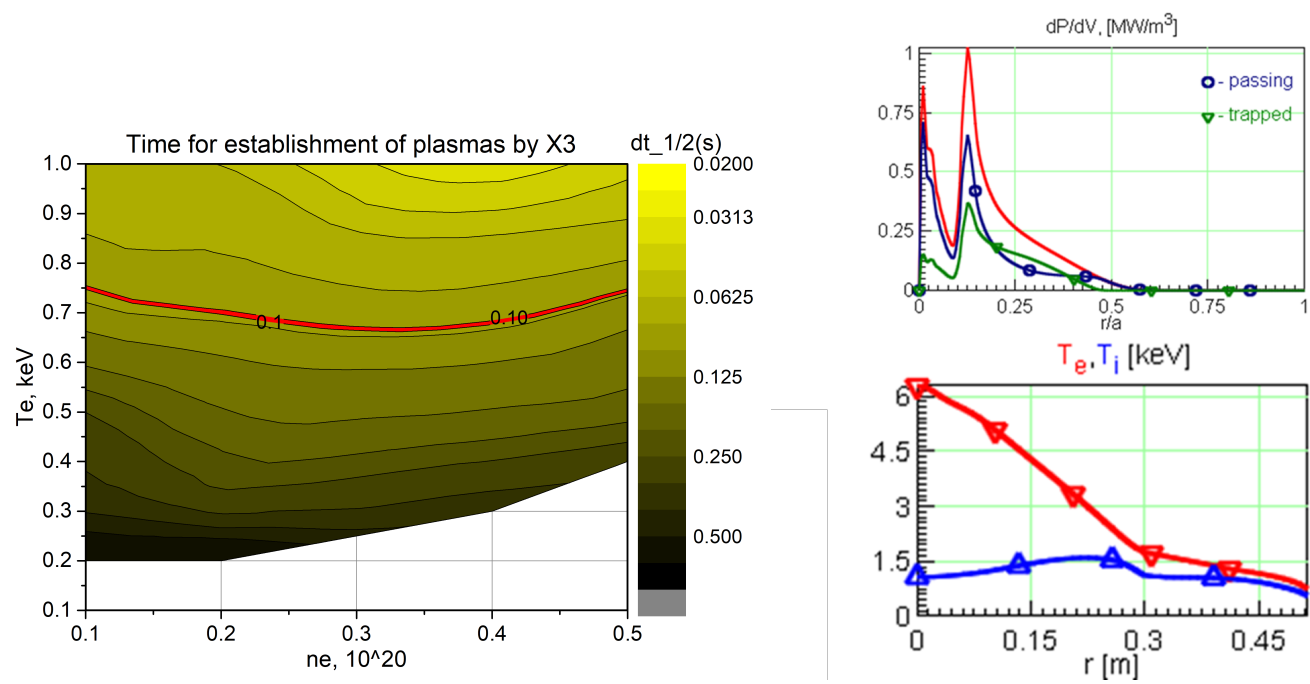

Figure 2. Left: The plot of $\Delta t_{1 / 2}$, i.e. the time interval required for evolution from the initial condition to the point where the half of launched power is absorbed; final steady state for plasmas with $5 \mathrm{MW}$ heating by X3-mode. The red line corresponds to the critical value $\Delta t_{1 / 2}=100 \mathrm{~ms}$. Right: For fixed density, $n_{e}(0)=3 \times 10^{19} \mathrm{~m}^{-3}$, the deposition profile, $d P_{\text {abs }} / d V$ (red for total, blue and green for passing and trapped electrons, respectively), and temperature profiles, $T_{e, i}$, are shown for the final steady state, $t \gg \Delta t_{1 / 2}$. The fractions of power absorbed by trapped and passing electrons are also shown.

which is reached for $n_{e}(0)=3 \times 10^{19} \mathrm{~m}^{-3}$ with $5 \mathrm{MW}$ of pure X3 heating. The solution obtained corresponds to the CERC (core electron root confinement) regime [13] which is characterized by large positive radial electric field, $E_{r}$, peaked $T_{e}$ and low $T_{i}$ due to a collisional decoupling of electrons and ions. One can find also that the integral absorbed power is about equally distributed between the trapped and passing electrons, which, potentially, might play a role for generation of non-local effects in the transport. Indeed, since the trapped electrons in stellarators, being locked within the toroidal mirrors, do not spread the absorbed power toroidally over the magnetic surface, the local absorbed power density is significantly increased (by a factor of five, at least), and some specific kinetic effects may be generated (additional convective losses [14], for example). Nevertheless, there is no clarity to date about the consequences of this effect.

The main conclusion from this consideration is the following: for the high-mirror magnetic configuration (UEM) with reduced magnetic field, $B_{0}=1.75 \mathrm{~T}$, target plasmas suited for X3 heating (a total power of $5 \mathrm{MW}$ was checked) must be preheated up to temperatures higher than $0.7 \mathrm{keV}$. This value has almost no dependence on the initial density. This requirement must be taken into account for any method used to create a target plasma for ECRH with the X3-mode.

\section{Target plasma with X2 (105 GHz) start-up}

In this section we consider possibilities to create the target plasmas with help of the X2-mode at $105 \mathrm{GHz}$. Again, while the gyrotrons can be used for generation of $105 \mathrm{GHz}$ without any problems (only the efficiency is reduced), the quasi-optical system needs to be tested and might require an additional optimization for this frequency. Below, we suppose that this option is available.

For simulations, we apply the same magnetic configuration and the same magnetic field, i.e. UEM with $B_{0}=1.75 \mathrm{~T}$. For X2 heating, four RF beams of $105 \mathrm{GHz}$ with the total power $2 \mathrm{MW}$ are considered. Since the cyclotron resonance is strongly shifted to the high-fieldside (HFS) and quasi-perpendicular launch seems to be rather ineffective (only plasma periphery can be heated), a moderate obliqueness for the beams needs to be used, $N_{\|} \simeq 0.25-0.3$. This case corresponds to off-axis heating at $r / a \simeq 0.5 \pm 0.2$ (see Fig.3). As expected for X2, the plasma is optically thick and the single-pass absorption is $100 \%$. Since the launch is oblique and located close to the maximum of $B$, only passing electrons absorb the power.

Transport simulations show that the temperature profiles established in steady-state are flat and $T_{e} \simeq T_{i} \simeq$ $1.4 \mathrm{keV}$, which is completely satisfactory for $\mathrm{X} 3$ operation. This scenario, as common with off-axis heating, is characterized by central "ion-root" confinement with negative radial electric field and with collisionally well coupled electrons and ions.

In addition to the steady-state simulations, one also needs to check the breakdown with RF heating beams. In W7-X, the typical time-delay between switch-on the power and plasma breakdown for quasi-perpendicular RF beams is about $10 \mathrm{~ms}$ [5]. However, since the energy of the seed electrons required for cyclotron interaction increases with obliqueness $\left(E_{0} \simeq m_{e} c^{2} N_{\|}^{2} / 2\right.$, as follows from the resonance condition), a similar tendency is expected for the time-delay, and, at first glance, oblique launch is not favorable for breakdown. At the same time, there are exper- 

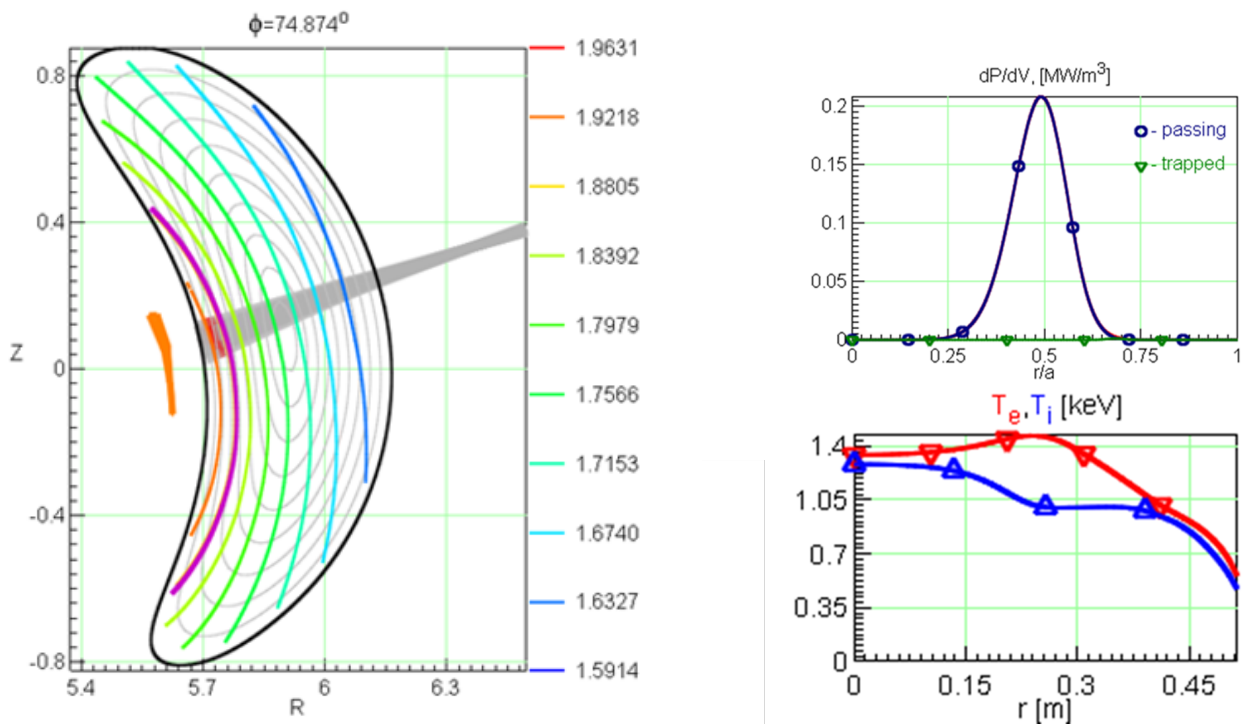

Figure 3. Left: $R-Z$ projection of the RF beam applied for $\mathrm{X} 2(105 \mathrm{GHz})$ heating. Right: Deposition profile, $d P_{\mathrm{abs}} / d V$, and temperature profiles, $T_{e, i}$, calculated for the same density as above, $n_{e}(0)=3 \times 10^{19} \mathrm{~m}^{-3}$. The results correspond to the final steady state.

imental investigations of the breakdown for both stellarators [16] and tokamaks [17] which confirm that its dependence on obliqueness is rather weak. Here, we assume that the time-delay for X2 breakdown is acceptable and focus only on steady-state parameters which must be compared with requirements for target plasmas.

Apart from the scenario considered for ECRH start-up, it seems realistic also to simplify the establishment of the initial plasma by using for the X2 breakdown (105 GHz) the field $B_{0}=1.87 \mathrm{~T}$. In this case, the location of the resonance is much more favorable and practically on-axis deposition can be obtained. As a 2nd step, a transition to parameters necessary for operation with the X3-mode needs to be done, i.e. ramping down the magnetic field while simultaneously increasing the obliqueness of the RF beams. Since the rate of change of the magnetic field is limited to $\left|d B_{0} / d t\right|<0.24 \mathrm{~T} / \mathrm{min}$, the time required for the transition from $1.87 \mathrm{~T}$ to $1.75 \mathrm{~T}$ can be estimated as $\Delta t>30 \mathrm{~s}$.

As a preliminary result from these considerations, one can conclude that establishment and preheating of target plasmas suited for stable X3-operation at reduced magnetic field, X2 ECRH start-up using beams with a frequency of $105 \mathrm{GHz}$ seems quite realistic.

\section{Summary}

For the magnetic configuration UEM characterized by very high mirror-ratio, $B_{01} / B_{00} \simeq 0.24$, the scenario with reduced magnetic field, $B_{0} \simeq 1.75 \mathrm{~T}$, was considered. Pure X3 heating at $140 \mathrm{GHz}$ was numerically investigated with help of the coupled ray-tracing code TRAVIS [10] and transport code NTSS [11]. General requirements for target-plasmas necessary for X3 operation were studied.

Despite incomplete single-pass absorption multipass $(\leqslant 3$ passes) heating allows deposition profiles well localized within the plasma core. The expected neoclassi- cal transport regime is CERC. At the same time, the X3mode alone appears to be insufficient for ECRH start-up and other methods need to be applied for creation of the target plasma with parameters necessary for X3 operation.

A scan over the initial electron density and temperature was performed for $6 \mathrm{RF}$ beams with $5 \mathrm{MW}$ total power. It was found that stable operation with the $\mathrm{X} 3$-mode is possible if the target plasma is preheated up to electron temperatures exceeding $0.7 \mathrm{keV}$ and this value has only a weak dependence on the initial density. If this condition is satisfied, the time interval required for evolution to the final steady-state with X3 heating does not take more than $100 \mathrm{~ms}$. This value meets with technical requirements to the safety of the mirrors used for beam reflection.

As an example of possible target-plasma creation for $\mathrm{X} 3$ operation, an X2 scenario at the frequency $105 \mathrm{GHz}$ was considered. Transport and ray-tracing simulations demonstrate that the temperatures in steady-state reach $T_{e} \simeq T_{i} \simeq 1.4 \mathrm{keV}$, be matching with the requirements found. This scenario is based on the assumption that the high obliqueness of the RF beams does not significantly reduce the breakdown efficiency. As an alternative, if breakdown far from the axis by oblique RF beams is problematic, the magnetic field $B_{0} \simeq 1.87 \mathrm{~T}$ for $\mathrm{X} 2$ breakdown at $105 \mathrm{GHz}$ can be applied since the cyclotron resonance is located close to the axis and no obliqueness required. In order to make a transition to X3 operation, the magnetic field needs to be ramped down (the required time is about of $30 \mathrm{~s})$.

As a preliminary result from this consideration, we can conclude that the establishment and preheating of target plasmas suitable for stable X3-operation at reduced magnetic field by X2 ECRH start-up using the RF beams at the optional frequency of $105 \mathrm{GHz}$ is quite realistic. Further options for target-plasma generation appear to be difficult (NBI) or need to be assessed (ICRH). 


\section{References}

[1] F. Warmer et al., Plasma Phys. Control. Fusion 58, 074006 (2016)

[2] J. Nührenberg and E. Strumberger, Contrib. Plasma Phys. 32, No. 3/4, 204 (1992)

[3] V. Erckmann et al., Fusion Sci. Technol. 52, 291 (2007)

[4] G. Dammertz et al., IEEE Trans. Plasma Sci. 34, 173 (2006) (https://ieeexplore.ieee.org/document/1621285)

[5] R. C. Wolf et al., Nucl. Fusion 57, 10202 (2017)

[6] K.J. Brunner et al., EPJ Web of Conferences 149, 03011 (2017) (https://doi.org/10.1051/epjconf/201714903011)

[7] A. Dinklage et al. Nature Physics 14, 855 (2018) (https://doi.org/10.1038/s41567-018-0141-9)
[8] Th. Rummel et al., IEEE Trans. Plasma Sci. 46, No. 5, 1517 (2018)

(https://ieeexplore.ieee.org/document/8327848)

[9] N. B. Marushchenko et al., EPJ Web of Conferences 87, 01007 (2015) (http://dx.doi.org/10.1051/epjconf/20158701007)

[10] N. B. Marushchenko, Y. Turkin, H. Maaßberg, Comp. Phys. Commun. 185, 165 (2014)

[11] Y. Turkin et al., Phys. Plasmas 18, 022505 (2011)

[12] S. P. Hirshman et al., Phys. Fluids 29, 2951 (1986)

[13] M. Yokoyama et al., Nucl. Fusion 47, 121 (2007)

[14] S. Murakami et al., J. Plasma Fusion Res. Ser. 1, 122 (1998).

[15] D. Gradic et al., Nucl. Fusion 55, 033002 (2015)

[16] K. Nagasaki et.al., Nucl. Fusion 45, 13 (2005)

[17] J. Stober et al., Nucl. Fusion 51, 083031 (2011) 